\title{
La Repubblica di Platone nell'esegesi simbolica, e metafisico-teologica di Proclo
}

\section{Plato's Republic in Proclus' symbolic and metaphysico-theological exegesis}

\author{
Michele ABBATE \\ Università degli Studi di Salerno \\ mabbate@unisa.it
}

Recibido: 16/05/2017 - Aceptado: 20/11/2017

DOI: https://doi.org/10.20318/fons.2017.3853

\begin{abstract}
Riassunto. Delle diverse tematiche affrontate da Proclo nella sua articolata interpretazione della Repubblica di Platone, il saggio propone una disamina di alcune fra quelle che appaiono particolarmente significative per comprendere in quale direzione proceda complessivamente l'esegesi procliana del dialogo platonico: lo бKomós (ossia l'argomento principale) e l'impianto simbolico della Repubblica (dissertazione I); la natura, la funzione e il ruolo della giustizia secondo l'esegesi procliana (dissertazioni III e VII-VIII); l'esame e la critica delle obiezioni mosse da Aristotele alla Repubblica di Platone (dissertazione XVII).
\end{abstract}

Parole chiave: Proclo, Platone, Aristotele, Repubblica, skopós, giustizia

\begin{abstract}
Among the various themes faced by Proclus in his articulated interpretation of Plato's Republic, this essay offers an examination of some of those that appear particularly significant in order to understand what direction Proclus' exegesis of this dialogue takes: the okomós (the main argument) and the symbolic system of the Republic (dissertation I); the nature, function and role of justice according to Proclus' exegesis (dissertations III and VII-VIII); the examination and criticism of the objections raised by Aristotle to Plato's Republic (dissertation XVII).
\end{abstract}

Keywords: Proclus, Plato, Aristotle, Republic, skopós, justice

Nell'ambito della tradizione filosofica neoplatonica, la Repubblica rappresenta certamente un testo fondamentale soprattutto in vista della complessiva sistematizzazione

ПНГН / F O N S II (2017), 153-176

ISSN 2445-2297 www.uc3m.es/pege
M. Abbate, La Repubblica nell'esegesi di Proclo DOI https://doi.org/10.20318/fons.2017.3853 
del pensiero platonico, alla quale la gran parte dei pensatori appartenenti a tale tradizione aspira. Un ruolo essenziale è attribuito dagli autori neoplatonici soprattutto alla metafora solare contenuta nel VI libro di questo dialogo platonico, ove, come noto, viene esposta l'analogia tra il Bene e il sole: la così detta "metafora solare" rappresenta un punto di riferimento esegetico essenziale per una delle tematiche fondamentali su cui si basa gran parte della riflessione metafisica neoplatonica, vale a dire l'identificazione dell'Uno, il Principio Primissimo, con il Bene'. D'altra parte la Repubblica, in considerazione della vastità e ampiezza delle questioni in essa affrontate, fornisce agli autori neoplatonici anche molteplici spunti di riflessione relativi a tematiche di varia natura, dall'etica all'ontologia, dalla teoria esegetica all'analisi dei differenti livelli entro cui risulta articolato il reale nel suo insieme, fino ad arrivare anche alla riflessione di tipo teologico che caratterizza in particolare la prospettiva neoplatonica di Proclo e la "Scuola (platonica) di Atene" di cui egli fu Diadoco.

Certamente è soprattutto in considerazione della sua vastità che quest'opera di Platone non poteva essere inserita nell'ordine canonico - stabilito originariamente all'interno della scuola giamblichea - dei dialoghi platonici che dovevano venire letti e commentati nella scuola ${ }^{2}$. La natura della Repubblica rendeva impossibile un commento sistematico, articolato e completo: troppo numerose e al contempo troppo ampie e complesse apparivano le tematiche ivi affrontate perché questo dialogo platonico potesse venire inserito tra le opere del curriculum formativo neoplatonico. Tuttavia ciò non significa ovviamente che esso venisse in qualche modo trascurato all'interno dell'intera tradizione neoplatonica, anzi sappiamo che la Repubblica, se non nella sua interezza, almeno in alcune sue parti ritenute più significative e centrali nella prospettiva esegetico-filosofica neoplatonica, è stata certamente oggetto di analisi puntuali e specifiche e quindi in varie forme commentata e discussa ${ }^{3}$. Ad ogni modo, tra i diversi testi antichi neoplatonici che avevano per oggetto specifico l'interpretazione della Repubblica è giunto sino a noi solo il così detto Commentario alla 'Repubblica' di Proclo, che risulta in realtà costituito da diciassette distinte dissertazioni, di varia natura e dedicate a tematiche specifiche che, più o meno direttamente, vengono sviluppate

${ }^{1}$ Su ciò si veda ABBATE 2016. Sulla centralità dell'interpretazione concernente la natura dell'Idea del Bene nella concezione plotiniana del Principio si veda inoltre GERSON 2016.

${ }^{2}$ Su tale questione si veda O’MEARA 1999. L'autore sottolinea peraltro come la non inclusione della Repubblica nel canonico ordine di lettura delle opere di Platone sia da attribuire a motivi di carattere pedagogico: «if the Republic was read in the Neoplatonic schools, why was it not included in the standard curriculum? The simplest answer to this would seem to be that it was simply for reasons of pedagogic convenience» (p. 194). Cfr. inoltre MANSFELD 1994, p. 34: «the Republic [...] does not belong with the ten dialogues of the canon of Iamblichus». Per un'analisi dettagliata del curriculum studiorum giamblicheo si veda anche O’MEARA 2003, pp. 61-68. Sul canone di lettura dei dialoghi platonici si veda inoltre Abbate 2012, in particolare pp. 27-30.

${ }^{3}$ Per una rassegna d'insieme degli autori neoplatonici che si occuparono, in varia misura, della Repubblica si vedano DörRIE, BALTES 1993, pp. 206-208. 
nel dialogo platonico ${ }^{4}$ : non si tratta, dunque, di un commento omogeneo, ma di un insieme di scritti raccolti in modo da formare un corpus di disparati testi esegetici accomunati dal fatto di essere tutti dedicati a questioni e tematiche affrontate nella $R e-$ pubblica ${ }^{5}$. Tale corpus esegetico, quindi, ci è pervenuto in una forma sostanzialmente diversa rispetto a quella degli altri commentari procliani, come quello all'Alcibiade I, al Timeo e al Parmenide che possono essere considerati come effettivi úmouvń $\mu \alpha \tau \alpha$, ossia "commenti" in cui le diverse parti del testo vengono direttamente citate e commentate in dettaglio. Delle diciassette dissertazioni che compongono il Commentario alla 'Repubblica' solo la XVI può essere considerata come un ampio e organico únó $\mu \nu \eta \mu \alpha$ al mito di Er nel X libro della Repubblica ${ }^{6}$. In ogni caso, oltre a questa raccolta di scritti di Proclo, noi non possediamo altre opere neoplatoniche interamente e specificamente dedicate a questo dialogo platonico. Si potrebbe dunque più esattamente parlare di Commentari procliani a parti e tematiche singole e specifiche trattate nella Repubblica. Tali testi, che in origine erano certamente separati e distinti tra loro e considerati tali dal nostro commentatore, sono stati con ogni probabilità raccolti successivamente in un'unica opera non dallo stesso Proclo, ma, verosimilmente, da qualche suo allievo o altro membro della scuola.

La particolare natura del non unitario e disomogeneo commentario procliano va certamente ricondotta proprio all'ampiezza e alla ricchezza delle tematiche affrontate nella Repubblica, senza dubbio considerata dagli autori neoplatonici come un'opera

${ }^{4}$ Per un'analisi articolata della natura dei diversi e disomogenei testi che formano questo commento procliano si può vedere ABBATE 2004, pp. XLV-LIV. Sullo stesso tema si rinvia inoltre al fondamentale studio di GallavotTi 1971. L'edizione di riferimento del Commentario alla 'Repubblica' è ancora quella in due volumi curata da KROLL 1899-1901.

${ }^{5}$ Tra le diverse dissertazioni che fanno parte del commentario procliano è opportuno segnalare che la VI è l'unica il cui argomento appare solo tangenzialmente riconducibile a temi concernenti la Repubblica. Tale testo, infatti, è un lungo ed assai ampio trattato autonomo, diviso in due libri ed articolato in vari capitoli. L'intero trattato potrebbe essere indicato con il titolo, riportato nel codice, del suo primo capitolo: Sulle considerazioni di Platone nella 'Repubblica' contro Omero e l'arte poetica, Kroll 18991901, I, pp. 69-205. Tale testo è stato con ogni probabilità inserito all'interno del commento poiché prende le mosse dalla critica mossa alla poesia da Platone nel III libro della Repubblica. In esso Proclo mostra come ciò che afferma Omero a proposito della dimensione divina risulta, se interpretato nella corretta prospettiva simbolico-allegorica, perfettamente in sintonia con la riflessione teologica di Platone. Secondo l'interpretazione di Proclo, in realtà Platone nella Repubblica condannerebbe la poesia omerica perché essa non è adatta all'educazione dei giovani: per comprendere i significati simbolici ed allegorici nascosti nelle opere omeriche per il nostro commentatore è necessaria, infatti, una imprescindibile e complessiva conoscenza teologica. L'autentico messaggio teologico di Omero, secondo Proclo, può essere colto solo se si è "iniziati". In questo modo il nostro commentatore riconduce ad una sostanziale armonia e concordanza la teologia platonica e la poesia omerica divinamente ispirata. Su questo trattato di Proclo e sul ruolo dell'interpretazione allegorica della poesia omerica nella speculazione teologica procliana si veda il volume di SHEPPARD 1980, in particolare pp. 145-161. Per l'uso della poesia omerica e per la sua interpretazione allegorico-simbolica in Proclo si veda PICHLER 2006, in particolare pp. 46-95.

${ }^{6}$ Tale testo occupa per grandissima parte il II volume di KROLL 1899-1901, pp. 96-359. 
sui generis che difficilmente poteva essere oggetto di un commento sistematico, esaustivo e completo da fissare nel curriculum della scuola.

Tra le diverse dissertazioni di questo corpus di scritti, è comunque possibile individuare un gruppo di testi che può venire considerato come il "commentario di base" dedicato da Proclo alla Repubblica, il quale costituisce una sorta di "introduzione" a questo dialogo platonico: si tratta delle dissertazioni I-V (la II è andata completamente perduta), VII-XII e XIV, nelle quali vengono prese in esame alcune delle problematiche fondamentali del dialogo ${ }^{7}$ : in particolare il suo tema principale congiuntamente all'analisi della sua struttura e impianto narrativo (dissertazione I), la concezione teologica (dissertazione IV e anche IX), quella etico-politica (dissertazioni III, VII-VIII) e quella relativa alla natura del filosofo (dissertazione X) che emergono da quest'opera platonica. Sono proprio queste le tematiche centrali affrontate da Proclo nella sua interpretazione, che devono essere esaminate per comprendere quale sia, in linea di massima, il senso complessivo dell'esegesi procliana insieme alla finalità che viene da lui attribuita a questo dialogo. A tali testi occorre aggiungere anche quanto viene affermato nella dissertazione XVII (così come altre dissertazioni di questo commentario, conservata solo parzialmente), la quale può essere intesa come una sorta di significativa appendice all'intero corpus, in quanto in essa vengono esaminate e criticate le obiezioni mosse da Aristotele nel II libro della Politica alla polis ideale di Platone.

Delle diverse tematiche affrontate da Proclo nella sua articolata interpretazione della Repubblica, intendo qui di seguito prenderne sinteticamente in esame alcune, le quali appaiono particolarmente significative per comprendere in quale direzione proceda l'esegesi procliana di questo dialogo 8 :

1. Lo бкотт́s (ossia l'“argomento principale") e l'impianto simbolico della Repubblica (dissertazione I);

2. La natura, la funzione e il ruolo della "giustizia" in questo dialogo secondo l'esegesi procliana (dissertazioni III e VII-VIII);

3. L'esame e la critica delle obiezioni mosse da Aristotele alla Repubblica di Platone (dissertazione XVII).

\footnotetext{
${ }^{7}$ Sulla dissertazione XI del commentario procliano, incentrata sull'interpretazione dell'analogia solare si può vedere ABBATE 1999; cfr. inoltre ABBATE 2003 e 2007.

${ }^{8}$ Per un'efficace e utile sintesi della prospettiva esegetico-filosofica di Proclo e delle strategie ermeneutiche da lui impiegate nell'affrontate le questioni fondamentali discusse nella Repubblica si veda VEGETTI 2009, pp. 34-38.
} 


\section{Lo бкото́s e l'impianto simbolico della Repubblica nell'interpretazione pro- cliana}

Nell'ambito della tradizione esegetica, propria specificamente del tardo neoplato-

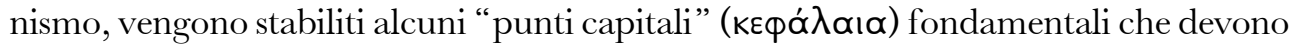
essere investigati prima di affrontare la lettura di ogni dialogo platonico o anche di altre opere filosofiche9: essi avrebbero permesso ai discenti, allievi della Scuola, di orientarsi in relazione all'argomento affrontato nell'opera che viene commentata dal maestro. L'insieme dei kєథá $\lambda \propto \_\alpha$ costituisce quella che potremmo definire a tutti gli effetti come una griglia ermeneutica fondamentale: i commenti dei testi filosofici, infatti, dovevano rispondere a precise domande e specifici criteri elaborati all'interno della Scuola. Tali criteri hanno una chiara funzione esegetica e didattica in quanto sono tesi ad individuare gli elementi strutturali di una determinata opera. Tra i diversi "punti capitali" che devono essere presi in esame prima della lettura di un'opera di Platone un ruolo assolutamente decisivo è attribuito allo бкото́s, letteralmente lo "scopo" o "obiettivo" principale, ossia il tema fondamentale del dialogo (o anche di qualsiasi altro testo filosofico) commentato. Insieme a questo, una fondamentale funzione nel caso specifico della Repubblica è riconosciuta all'intreccio narrativo e alla particolare ambientazione del dialogo.

Relativamente alla trattazione dei кєфá $\lambda \propto \ltimes \alpha$, la Repubblica sembra venire considerata da Proclo come un testo assolutamente esemplare in grado di fornire una sorta di riferimento paradigmatico per l'esegesi di tutti i dialoghi platonici. Proprio all'inizio della I Dissertazione, infatti, il nostro commentatore, proponendosi di illustrare come

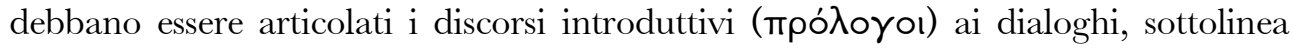
come la Repubblica rappresenti un "banco di prova" per l'individuazione delle questioni preliminari che costituiscono le linee portanti dell'esegesi dei testi platonici ${ }^{10}$.

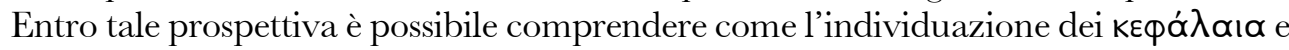

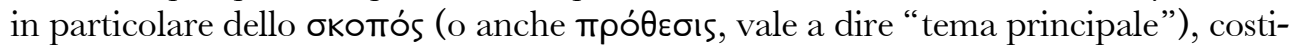
tuisca per gli interpreti neoplatonici uno schema isagogico che permette di inquadrare le diverse opere commentate sulla base del loro specifico oggetto e della loro struttura argomentativa. Infatti è proprio lo бKomós a costituire il fulcro dello schema isagogico

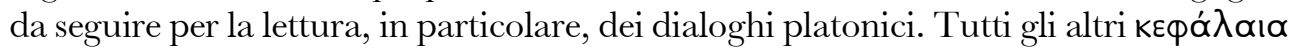
devono, in qualche modo, essere orientati e convergere nella direzione dello бKoтós e, quindi, essere in perfetto accordo con quest'ultimo. Come sottolinea Proclo ancora all'inizio della I dissertazione, i discepoli dovranno seguire questo schema di fondo

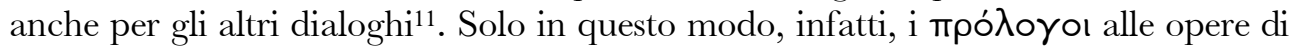

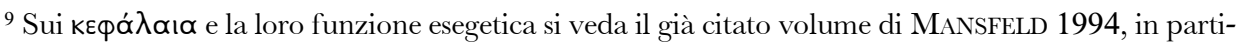
colare pp. 30-37. Si veda inoltre ABBATE 2012, in particolare pp. 84-87; per quanto riguarda specificamente il Commento alla Repubblica si veda ancora ABBATE 2004, in particolare pp. LXIV-LXX.

${ }^{10} \mathrm{Su}$ ciò cfr. Commento alla 'Repubblica' [d'ora in poi In R.], dissertazione I, vol. I, p. 5, 6 segg.

${ }^{11}$ Cfr. In. R., I, p. 5, 9-12, ove, in sostanza, Proclo afferma che gli allievi devono seguire le indicazioni
} 
Platone risulteranno completi, rivelando la struttura interna dei dialoghi ed il loro significato di fondo. Nella Repubblica in effetti, precisa ancora il nostro commentatore, la centralità dello бкото́s riveste un valore assolutamente paradigmatico: in tale dialogo, infatti, è possibile cogliere in modo chiaro e perspicuo come i tutti "punti capitali" nella loro struttura d'insieme risultino convergere armonicamente verso un unico e medesimo "argomento/obiettivo di fondo" (бкото́s) ${ }^{12}$.

Qual è, dunque, per Proclo il tema fondamentale, lo бкото́s della Repubblica?

Come egli stesso osserva affrontando tale questione, v'è un profondo disaccordo

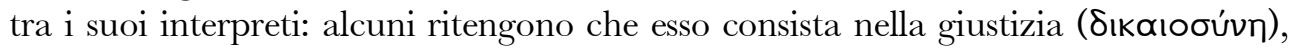
altri invece, sottolineando che la discussione concernente la giustizia è preliminare e introduttiva, considerano come suo principale obiettivo/argomento la "forma di governo" /"costituzione" (то入ıтвía) ${ }^{13}$. La soluzione proposta da Proclo è sostanzialmente di compromesso: in quanto sia la giustizia sia la forma di governo rappresentano i due argomenti fondamentali del dialogo ${ }^{14}$. Essi, d'altra parte, convergono e vengono a coincidere in un unico e medesimo obiettivo d'insieme: non si tratta dunque di due okotroí effettivamente distinti, bensì essi vanno considerati come coincidenti tra loro $^{15}$. Come infatti spiega Proclo, in una medesima opera non ci possono essere due differenti scopi, ma l'argomentazione (ó $\lambda$ ó $\gamma \circ \varsigma$ ) deve risultare simile ad un essere vivente ( $\zeta \omega \omega)$, perfettamente armonica e coordinata in tutte le sue parti in base

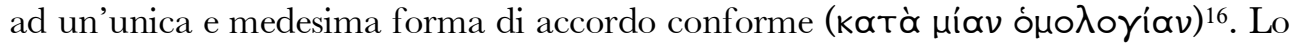
бKoтtós, dunque, nel microcosmo del dialogo rinvia per analogia alla strutturale unità

del maestro come delle tracce (' $(\mathbf{x} \cup \eta)$; essi, inoltre, dovranno seguire le medesime linee esegetiche anche per gli altri dialoghi. Si tratta di una chiara, precisa e consapevole indicazione di metodo. Nell'introduzione all'edizione dello scritto anonimo Prolegomeni alla filosofia di Platone WESTERINK 1990 ha dimostrato che i capitoli II-XI di quest'opera risalgono probabilmente ad un'introduzione alla filosofia di Platone scritta da Proclo. Tali capitoli contengono i dieci punti canonici in cui era articolato lo schema isagogico alla filosofia di Platone. Per un chiaro e preciso commento di quest'opera si rinvia al recente volume, con saggio introduttivo, curato da MotTA 2014. Circa l'ipotesi che sia stato Proclo a dare un ordinamento canonico agli schemi introduttivi delle opere di Platone ed anche a quelle di Aristotele schemi che ritroviamo, in effetti, anche nei commentatori posteriori - si veda HADOT 1987.

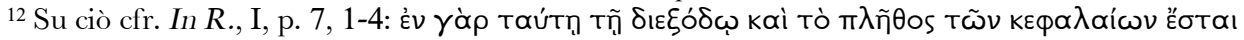

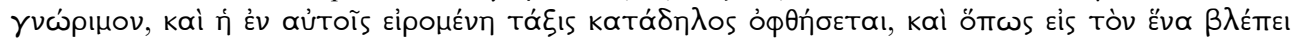

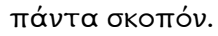

${ }^{13}$ Cfr. In R., I, p. 7, 5-11, 4.

14 Su ciò ci veda MANSFELD 1994, pp. 31-33.

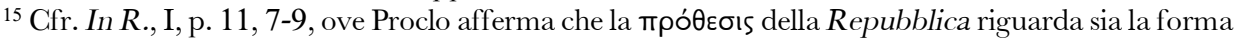

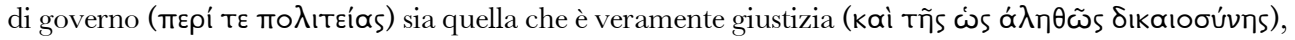

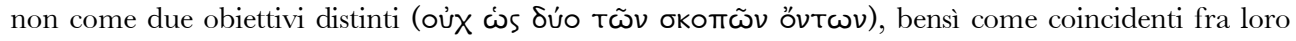

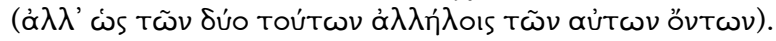

${ }^{16} \mathrm{Su}$ ciò cfr. In R., I, p. 7, 9-12. 
del macrocosmo costituito dal reale ${ }^{17}$, la cui integrità d'insieme, nella prospettiva neoplatonica procliana, è garantita dal suo fondamento originario, ossia l'Uno-Bene. Analogamente a quest'ultimo, lo бкото́s rappresenta il fulcro e il fattore unificante dell'intera struttura dell'opera: nel caso specifico della Repubblica, esso è dunque rappresentato al contempo dalla riflessione sulla giustizia intrinsecamente congiunta a quella sulla forma di governo. Si comprende allora come questo dialogo, nella sua strutturale ed al contempo intrinsecamente unitaria complessità e ampiezza, rappresentasse per Proclo un significativo esempio di come la coerenza e armonia di un testo sia garantita dalla funzione unitaria e unificante svolta dallo бкотós. In effetti, se si considera il complessivo impianto argomentativo di questo dialogo, esso pare svilupparsi proprio intorno alla riflessione sulla giustizia alla quale è intrinsecamente connessa quella riguardante la forma di governo ideale che deve costituire la struttura

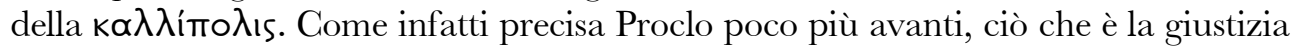
in una singola anima, lo stesso è, certamente, nella città ben governata la sua specifica forma di governo ${ }^{18}$. È proprio in questa prospettiva, del resto, che Proclo interpreta il senso fondamentale dell'analogia fra parti dell'anima e struttura tripartita della città ideale nella Repubblica ${ }^{19}$ : un'analogia che agli occhi di Proclo non è solo argomentativa e, per così dire, concettuale, bensì anche assolutamente concreta ed effettiva, in quanto rinvia alla strutturale analogia che connette fra loro i vari livelli entro cui si articola tutto il reale nella sua interezza e complessità.

A fornire un ulteriore argomento a sostegno della perfetta coerenza, unitarietà e consequenzialità argomentativa della Repubblica, è, in base all'esegesi procliana, anche l'impianto narrativo e simbolico che permea l'intero dialogo. In effetti, se si considera che secondo gli esegeti appartenenti alla tradizione tardo-neoplatonica la comprensione dei codici allegorici e simbolici dei dialoghi è alla base di una corretta interpretazione delle dottrine platoniche, è possibile comprendere come la Repubblica, in modo sempre esemplare agli occhi di Proclo, con la sua particolare ambientazione e il suo articolato impianto narrativo si offra ad un'unitaria interpretazione simbolica che si fonda su una particolare lettura allegorica del luogo e delle circostanze specifiche che vengono indicati proprio all'inizio del I libro del dialogo platonico. Secondo Proclo, l'impianto simbolico-narrativo che sorregge la Repubblica appare incentrato proprio sulla particolare ambientazione del dialogo, alla quale Platone accenna già con le primissime parole del I libro con le quali Socrate inizia la narrazione della discussione da lui sostenuta al Pireo ${ }^{20}$. Il porto di Atene è metaforicamente inteso da

\footnotetext{
${ }^{17}$ Sull'analogia tra macrocosmo reale e microcosmo testuale nell'esegesi del tardo neoplatonismo, si veda l'ancora fondamentale volume di COULTER 1976.

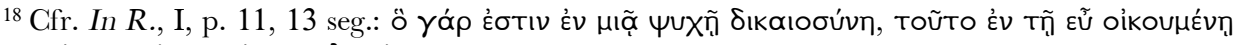

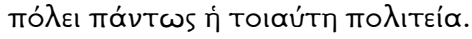

${ }^{19} \mathrm{Su}$ ciò cfr. In R., I, p. 11, 13 segg.

${ }^{20}$ Su ciò cfr. Platone, Repubblica I, 327a1, ossia le parole con cui Socrate inizia la propria narrazione
} 
Proclo come luogo simbolico e dimensione della confusione e dell'eterogeneità oltre che dell'incertezza e dell'instabilità proprie del mondo fenomenico. In questa dimensione di incertezza e confusione, simbolizzate, secondo quanto afferma Proclo, nei dialoghi platonici da tutto ciò che si trova nei pressi del mare ${ }^{21}$, Socrate, all'inizio della Repubblica, è costretto a combattere contro quella figura che, invece, è perfettamente a proprio agio nella dimensione in cui regnano l'incertezza e la confusione: è il sofista, al quale Proclo sembra attribuire una sorta di consistenza metafisica, come se si trattasse di un particolare livello ipostatico del reale; in questo contesto, infatti, il nostro commentatore afferma che Socrate lotta in difesa della giustizia (ưrtè Tñs

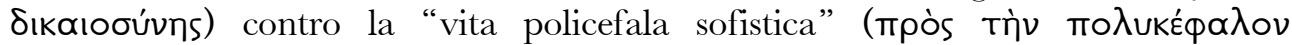

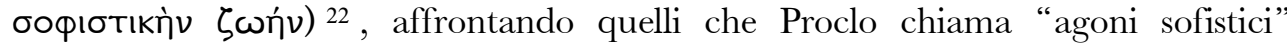
(бофıбтікоі ớ $\gamma \tilde{\omega} v \varepsilon \varsigma)^{23}$. La "vita policefala sofistica" è quella che caratterizza la dimensione simbolizzata dal Pireo, nella quale regnano la confusione, l'incertezza e lo smarrimento epistemico, al quale Socrate sembra soccombere per quasi tutto il I libro della Repubblica. Il riferimento è ovviamente al sofista Trasimaco, sostenitore di un radicale nichilismo etico, contro il quale Socrate, appunto nel I libro, deve fare i conti per poter difendere la propria concezione eticamente positiva della giustizia. Trasimaco viene così a simbolizzare per Proclo la natura particolare del sofista, ossia di quell'essere proteiforme che, come viene detto nel Sofista, si difende nascondendosi e attacca rendendosi inafferrabile e sfuggente. Come le teste dell'Idra, gli argomenti del sofista, "custode" della dimensione salmastra e confusa del Pireo ${ }^{24}$, sembrano così capaci di rigenerarsi e attaccare ogni argomentazione che non risulti poggiare su basi teoretiche sicure: lo scontro con il sofista nel I libro della Repubblica sembra in effetti assumere il carattere della disputa argomentativa nella quale si viene catapultati in un labirinto epistemico dal quale non sembra possibile trovare una via d'uscita. Il Pireo

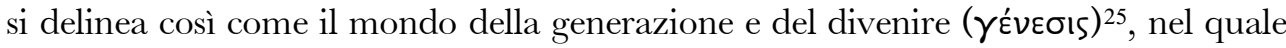
nulla rimane stabile, ma tutto viene trascinato alla deriva in un'incessante fluire: nel mare gonfio di tempesta del divenire anche le anime che vivono secondo ragione, come Socrate, corrono il pericolo di venire sommerse e travolte dalle ondate più pericolose $^{26}$. Proclo in questo modo sembra cogliere uno degli aspetti assolutamente decisivi per comprendere la particolare funzione che il I libro riveste nell'impianto generale di questo dialogo: l'iniziale difficoltà e incertezza in cui sembra trovarsi Socrate

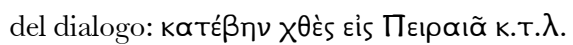

${ }^{21}$ Su ciò cfr. In R., I, p. 17, 3-5.

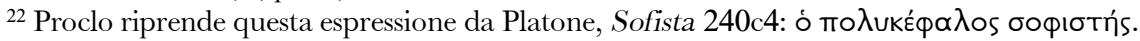

${ }^{23}$ Cfr. In R., I, p. 17, 17-19.

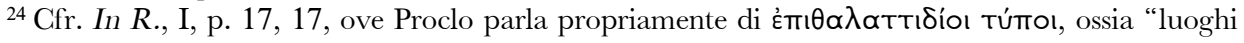
sulla riva/in prossimità del mare".

${ }^{25}$ Cfr. In R., I, p. 17, 23.

${ }^{26} \mathrm{Su}$ ciò cfr. In R., I, p. 17, 25 segg. 
di fronte alle argomentazioni di Trasimaco la cui tesi principale è, come noto, che "il giusto è l'utile del più forte". Del resto, è proprio in risposta a tale affermazione che si sviluppa l'intero dialogo, in tutti i suoi dieci libri.

Nell'interpretazione procliana, anche la circostanza specifica nella quale avviene la discussione narrata nella Repubblica, acquista un particolare e centrale significato simbolico-allegorico: tale circostanza è costituita dalla celebrazione delle feste rituali dedicate alla dea Bendis ${ }^{27}$. In effetti, da ciò che Proclo afferma a proposito del luogo in cui è ambientato il dialogo, sembra emergere, in modo implicito, una decisiva domanda: per quale ragione Socrate è disceso in questa dimensione instabile ed in continuo mutamento, all'interno della quale si corre il rischio di venire travolti ed essere spinti alla deriva? La risposta a questa implicita domanda si cela nell'articolata interpretazione simbolico-allegorica della circostanza specifica in cui avviene il dialogo della Repubblica.

L'inizio del I libro, in effetti, è per gran parte incentrato sulla festa in onore della misteriosa dea tracia Bendis, festa che si tiene al Pireo. Socrate è spinto a rimanere proprio dalla curiosità che in lui suscita tale festa. Anche in considerazione di ciò, Proclo dà grande importanza alla circostanza specifica del dialogo, ossia le Bendidie, la festa in onore di Bendis, interpretate dal nostro commentatore in connessione con la festa delle Panatenee cui si accenna nel Timeo e che rappresenta la circostanza specifica di tale dialogo. Non è qui possibile affrontare in dettaglio in tutte le sue articolazioni la complessa interpretazione simbolica proposta da Proclo, per il quale, occorre comunque precisare, la circostanza specifica nella Repubblica ${ }^{28}$, le Bendidie appunto, appare in perfetto accordo con il luogo, il Pireo, in cui si svolge il dialogo ${ }^{29}$. Pertanto il nostro commentatore si sofferma a considerare la natura della dea Bendis: essa sarebbe il corrispettivo tracio di Artemide ed inoltre "Bendis", continua Proclo, è uno dei molti nomi che il teologo trace Orfeo attribuisce alla dea Selene ${ }^{30}$. In considerazione di ciò, Bendis sembrerebbe rappresentare, secondo l'interpretazione procliana, una divinità encosmica che fornisce luce, anche se tenue e non splendente come quella del sole, alla dimensione fenomenica. Entro tale prospettiva, Bendis si delinea come la dea, per così dire, simbolo della dimensione di cui fa parte il Pireo: luogo emblematico dello smarrimento epistemico, ma nel quale è possibile trovare un orientamento attraverso la discussione dialettica e la riflessione filosofica. Perciò Proclo afferma che nell'incontro del Pireo l'anima viene raffigurata nell'atto di dare un ordine, seppure non senza difficoltà e confusione, alla dimensione stessa del divenire ${ }^{31}$.

${ }^{27} \mathrm{Su}$ ciò cfr. Platone, Repubblica, I 327a2 segg.

${ }^{28}$ Per un'analisi dettagliata dell'interpretazione simbolica della circostanza specifica della Repubblica cfr. ABBATE 2004, pp. LXX-LXXXVII.

${ }^{29}$ Su ciò cfr. In R., I, p. 18, 7 segg.

${ }^{30}$ Cfr. In R., I, p. 18, 10-16.

${ }^{31} \mathrm{Su}$ ciò cfr. In R., I, p. 19, 3 segg. 
Questo sembra in conclusione il senso complessivo dell'interpretazione procliana del luogo e della circostanza specifica del dialogo: Socrate deve discendere nella dimensione chiaroscurale simbolizzata dal Pireo, regno del sofista, per trovare la via d'uscita dall'intricato labirinto del divenire e dall'iniziale smarrimento epistemico dovuto alle argomentazioni sofistiche di Trasimaco. In tal modo il filosofo, dopo aver affrontato il sofista e avendolo sconfitto sul suo stesso terreno, potrà trovare il proprio orientamento, comprendendo in quale direzione egli debba volgere la sua ricerca. La

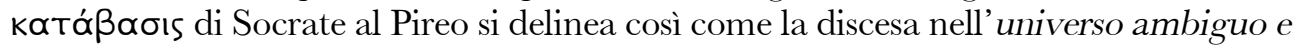
oscuro di Bendis, donde l'indagine filosofica deve prendere le mosse per potersi trasformare da paralizzante contesa eristica in autentica impresa dialettica volta a raggiungere, in ultima istanza, quel fondamento epistemologico ultimo che è in grado di dar luce ad ogni reale ed autentica conoscenza.

2. Natura, funzione e ruolo della "giustizia" nella Repubblica secondo l'esegesi procliana: l'armonia della città come imitazione dell'armonia divina del Tutto

Nella VII dissertazione vengono prese in esame le teorie, esposte nel IV libro della Repubblica, sulla tripartizione dell'anima e sulle sue virtù. In questo testo Proclo, riprendendo la concezione aristotelica contenuta soprattutto nel I libro dell'Etica Nicomachea, precisa quale sia l'effettiva natura della "virtù" (àpetŕ): essa è innanzitutto ciò che rende perfetta la condizione dell'elemento cui essa inerisce. Proclo, tuttavia, precisa che la "virtù" in senso proprio è quella che ha a che fare con la vita: infatti l'autentica virtù è "perfezionatrice" della vita cui essa appartiene: per suo effetto chi la possiede è nella condizione di esercitare al meglio le proprie potenzialità ${ }^{32}$. Inoltre, precisa il nostro commentatore, si deve distinguere la perfezione e l'attività di un essere considerato in se stesso rispetto alla perfezione e all'attività del medesimo essere considerato in relazione con altro.

Tale presupposto è per Proclo decisivo per l'esame della concezione platonica relativa alle tre parti dell'anima e ai tre generi politici che costituiscono l'insieme della città. Sia l'anima che la città, così come ogni altra realtà particolare che, in quanto tale, è soggetta ad un'intrinseca molteplicità e divisione, devono venire a formare unità organiche: di conseguenza, l'analisi delle loro rispettive perfezioni ed attività deve tener necessariamente conto soprattutto del rapporto reciproco, per quel che concerne l'anima, tra le sue diverse componenti e, per quel che concerne la città, tra i diversi generi politici. Infatti è solo in virtù di questa relazione reciproca tra le loro diverse parti che si può realizzare l'armonia all'interno dell'anima e all'interno della città.

Proclo passa allora a considerare il ruolo che nell'anima deve ricoprire la parte razionale, la quale ha in essa il compito di governare le componenti irrazionali: la sua

${ }^{32} \mathrm{Su}$ ciò cfr. In R., I, dissertazione VII, p. 206, 10-16. 
attività dunque va posta necessariamente in relazione alle altre parti dell'anima. Di conseguenza l'attività della componente razionale che è in noi non può essere limitata alla mera contemplazione, che pure costituisce il suo fondamentale compito in considerazione della sua propria essenza, poiché la sua funzione all'interno dell'anima è quella di governare ${ }^{33}$. Se si limita alla pura attività contemplativa, la parte razionale viene meno al compito che essa deve svolgere in relazione alle altre parti. Infatti compito di chi governa è quello di procurare a ciò che è governato il bene. Disattendendo a questo compito, che consiste nel garantire il bene a tutto l'insieme dell'anima, essa finirebbe per incrinare la necessaria unità organica delle parti con il tutto ${ }^{34}$. Si potrebbe dire dunque, facendo riferimento alla concezione metafisica neoplatonica di Proclo, che nell'elemento razionale, nel caso in cui esso si limiti all'attività contemplativa e si disinteressi del bene delle altre parti, prevarrebbe la sua natura di realtà particolare e parziale, il che impedirebbe all'anima nel suo complesso di costituire un tutto unitario e organico. Tuttavia anche le altre componenti dell'anima, se agissero in modo assolutamente autonomo e non venissero dirette in relazione alla funzione che esse devono svolgere rispetto alle altre, finirebbero per essere causa di divisione e disgregazione per la totalità unitaria dell'anima ${ }^{35}$

Nell'anima, dunque, le virtù delle sue tre componenti, che sono necessariamente collegate fra loro, costituendo un'unica e medesima vita ( $\mu$ ía $\zeta \omega \eta ́$ ), vanno considerate

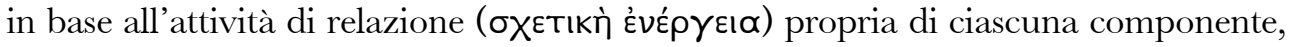
e nella stessa prospettiva si deve considerare anche il vizio (kakía), il quale si configura così come quella condizione che distrugge la reciproca relazione vitale conforme a

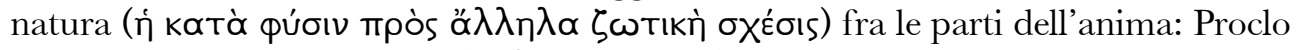

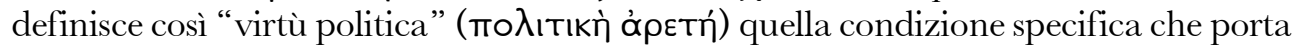

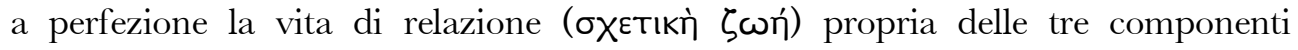
dell'anima, mentre il vizio rappresenta la condizione opposta a questa ${ }^{36}$.

Entro tale prospettiva, la virtù politica viene così intesa come la fonte e la garanzia dell'armonia e dell'unità interna dell'anima. A un primo livello essa sembra venir

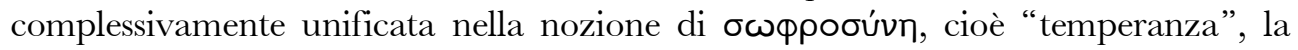
quale, estendendosi a tutte e tre le parti e facendo sì che ciascuna svolga solo quel preciso compito che ad essa è assegnato in vista del bene di tutto l'insieme, fornisce

${ }^{33}$ Cfr. In R., I, p. 208, 5 segg., ove viene messo in luce come la parte razionale, se viene considerata in se stessa e non in rapporto alle altre, svolga il suo compito specifico attraverso la pura attività contemplativa; infatti tale attività nell'ambito dell'anima è conforme alla specifica natura ed essenza della componente razionale. D’altra parte, poiché l'anima razionale ha pure il compito di governare le altre componenti, essa deve essere considerata anche in rapporto a tale attività di relazione.

${ }^{34}$ Cfr. In R., I, p. 208, 11 segg.

${ }^{35} \mathrm{Su}$ ciò cfr. In R., I, p. 208, 14 segg.

${ }^{36}$ Cfr. In R., I, pp. 208, 25-209, 2. 
un'armonia complessiva ${ }^{37}$. D'altra parte è necessaria e fondamentale un'ulteriore virtù con la specifica funzione di mantenere e conservare l'accordo e l'armonia complessiva e unitaria delle tre parti. Proprio in questo senso Proclo interpreta la funzione

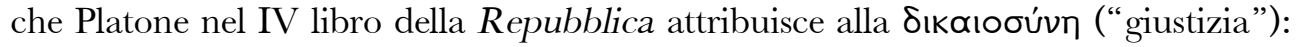
tale virtù, afferma Proclo, attiene a tutte le parti e fa sì che ciascuna agisca sulla base

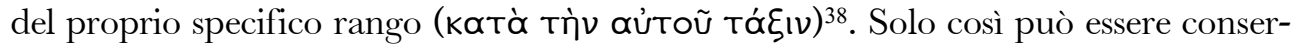
vata e mantenuta la relazione armonica e reciproca tra le parti: è proprio grazie a essa che ogni singola anima, benché sia una realtà particolare ed in quanto tale intrinsecamente soggetta alla divisione, viene a costituire un'unità complessiva e organica.

Occorre a questo punto prendere in esame la prospettiva entro la quale Proclo interpreta la concezione platonica relativa al rapporto tra le parti dell'anima ed i ge-

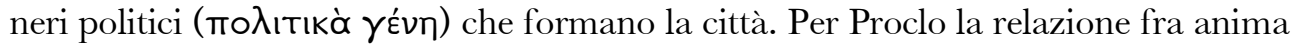
e città non è semplicemente analogica, ma come egli afferma esplicitamente, le virtù o i vizi delle parti dell'anima preesistono come paradigmi ( $\pi \propto \rho \alpha \delta \varepsilon i ́ \gamma \mu \alpha \tau \alpha)$ alle virtù o ai vizi insiti nei generi politici ${ }^{39}$. In effetti, secondo la prospettiva neoplatonica procliana, rispetto alla città l'anima appartiene a un livello di realtà entro cui regna una maggiore semplicità: si deve, infatti, tenere presente che, secondo uno dei principi e presupposti concettuali fondamentali dell'intera riflessione metafisica neoplatonica, le realtà più semplici appartengono ad un livello superiore rispetto a quelle più complesse e caratterizzate da un livello di molteplicità maggiore. Così, nell'interpretazione procliana del rapporto fra anima e città è possibile intravedere quella concezione di base propria, in particolare, del tardo neoplatonismo, secondo cui il Tutto, pur essendo in se stesso articolatamente molteplice, costituisce comunque un insieme organico e un'unità perfettamente armonica sempre in virtù del fondamento supremo della totalità unitaria del reale, l'Uno-Bene.

Secondo Proclo, dunque, l'anima, che è alla base della ben più complessa e articolatamente molteplice dimensione costituita dalla città, rappresenta il modello rispetto al quale l'insieme della città è l'immagine. Di conseguenza, conformemente a tale

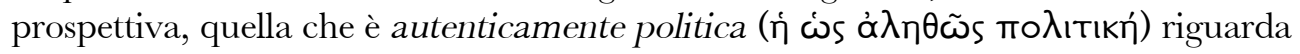
le parti dell'anima, mentre quella che appartiene ai generi politici deve essere a sua volta considerata come immagine ( $\varepsilon i k \omega ́ v)$ dell'autentica politica ${ }^{40}$. Si viene così a configurare una generale simmetria fra i vari piani entro i quali è articolata la struttura complessiva dell'intero cosmo: in base all'esegesi procliana, tra le diverse parti dell'anima e tra i diversi generi politici che costituiscono nel suo insieme la città si deve

${ }^{37}$ Su ciò cfr. In R., I, p. 212, 11-26. Per un'analisi più dettagliata di quanto viene affermato in questo passo cfr. ABBATE 2006, in particolare pp. $188 \mathrm{segg}$.

${ }^{38}$ Cfr. In R., I, p. 214, 3-7.

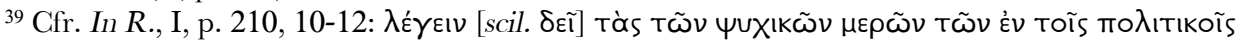

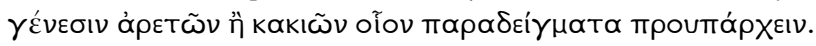

${ }^{40} \mathrm{Su}$ ciò cfr. In R., I, p. 210, 27-30. 
rispecchiare quella più generale armonia che pervade il Tutto. Dunque, in ultima analisi, come l'anima, anche la città, per divenire, pur nelle sue molteplici componenti, un'unità autenticamente armonica, deve rispecchiare nella sua struttura l'ordine del cosmo, che, a sua volta, deriva e dipende dai livelli più alti del reale: questi ultimi, sulla base della complessiva teologizzazione della realtà ${ }^{41}$ elaborata da Proclo, corrispondono a specifici livelli divini.

Di conseguenza, alla luce di quanto sin qui si è detto, si potrebbe sintetizzare la prospettiva esegetica di Proclo riguardante la tripartizione dell'anima e le tre classi che formano la città con l'espressione cui egli ricorre in un passo della VIII Dissertazione: «questa è la cosa veramente più utile per le parti, imitare il Tutto, e per le forme di governo umane, divenire simili a quelle divine» ${ }^{42}$.

Secondo Proclo, in effetti, anche dal punto di vista della dottrina teologica platonica l'anima implica in se stessa un originario legame con i livelli divini più elevati del reale. Per dimostrare come l'anima, benché tripartita, contenga in se stessa un principio di unità e armonia, Proclo si rifà alla dottrina esposta da Platone nel Timeo, secondo la quale le componenti irrazionali sono introdotte dagli "dèi giovani" 43 , che per Proclo corrispondono agli dèi encosmici, mentre la ragione, che rappresenta la componente immortale dell'anima, è introdotta dal solo e unico Demiurgo ${ }^{44}$, identificato dal nostro commentatore con lo Zeus intellettivo ${ }^{45}$. Di conseguenza, il Demiurgo è la causa autenticamente originaria della totalità dell'anima, in quanto è da questo dio che dipendono anche gli dèi giovani ai quali è demandato il compito di plasmare i corpi mortali e con essi le componenti irrazionali dell'anima; inoltre sempre il Demiurgo, fonte dell'ordine e dell'armonia universali che permeano il cosmo, risulta, in ultima istanza, anche il fondamento dell'armonica unificazione delle parti dell'anima.

Come risulta evidente da queste ultime considerazioni, anche in riferimento alla tripartizione dell'anima e ai tre generi politici che costituiscono la città, Proclo trova il modo per ricondurre la questione all'ambito della riflessione teologica.

A questo punto appare inevitabile chiedersi quali possano essere allora, entro la prospettiva ermeneutica procliana, il ruolo e la funzione attribuiti alla figura del filosofo-guardiano. Anche l'attività di quest'ultimo, così come quella della parte razionale dell'anima, se viene considerato in se stesso e non in relazione agli altri individui o ai generi politici che costituiscono la città, è confinata alla pura contemplazione:

${ }^{41}$ Sul concetto di “teologizzazione del reale” in Proclo si può vedere ABBATE 2008, in particolare pp. 11 segg.

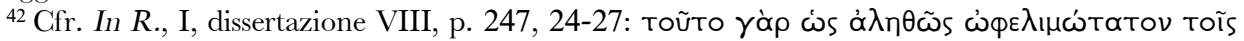

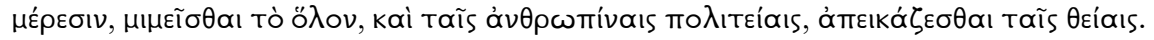

${ }^{43}$ Cfr. Platone, Timeo in particolare $42 \mathrm{~d} 2 \mathrm{segg}$.

${ }^{44}$ Cfr. In R., I, dissertazione VII, p. 234, 24-26.

${ }^{45}$ Sulla natura intellettiva di Zeus Demiurgo e sulla sua funzione all'interno della prima triade intellettiva cfr. ancora ABBATE 2008, pp. 129-134. 
egli, si potrebbe dire, contempla le realtà intelligibili e alla luce di queste giunge ad elevarsi fino al Bene. Ma l'attività del filosofo-guardiano non può limitarsi a ciò, altrimenti egli viene meno a quel ruolo di governo che gli è affidato ${ }^{46}$. Questo è il motivo per cui Socrate non permette che costui si limiti alle vette della vita contemplativa, ma lo fa ridiscendere da queste affinché si prenda cura della città. Solo se si dedica, oltre che alla riflessione filosofica, alla vita di governo e dunque all'attività di relazione che gli compete, egli può essere considerato autenticamente guardiano ${ }^{47}$.

Il filosofo-guardiano appare così fungere da intermediario fra la dimensione intelligibile e il mondo sensibile. La sua attività di governo si realizza come un prendersi cura della città in modo tale che essa rifletta, a livello di immagine, l'ordine e l'armonia che regnano nei livelli più alti della realtà. Egli risulta così in grado di rendere la città, che è pur sempre intrinsecamente molteplice e corruttibile, un'unità organica nella cui armonia viene rispecchiata la perfetta e divina organizzazione che governa e permea l'universo.

Il versante teologico appare, a più riprese, decisivo nell'esegesi procliana della $R e$ pubblica e in questo senso il nostro commentatore interpreta anche quanto viene affermato in un noto passo con cui si conclude il libro IX libro del dialogo: se, come

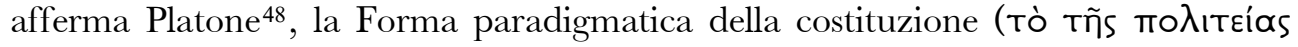

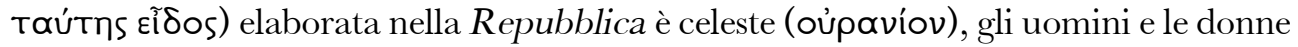
devono imitare, secondo Proclo, gli esseri divini ${ }^{49}$. Ciò sarà possibile solo una volta che tanto gli uomini quanto le donne siano stati predisposti ad imitare attraverso l'opportuna educazione quell'ordine e quell'armonia che regnano nella dimensione divina. Nella prospettiva procliana, infatti, se, come si è detto ${ }^{50}$, la cosa assolutamente più utile e giovevole per le parti è imitare il Tutto, allora ne consegue necessariamente che per le forme di governo umane la cosa più utile e giovevole sia rendersi simili a quelle divine.

Con una sfumatura di matrice teologica Proclo interpreta anche la concezione di Platone sull'uguaglianza di educazione e compiti per uomini e donne nella città ideale. L'armonia dell'intera città, afferma infatti il nostro commentatore, sarà maggiore se in essa vi sarà uniformità e se in tutti gli individui che la compongono, uomini e donne, regnerà la virtù: infatti, conclude Proclo, il Padre di questo nostro universo, ossia il Demiurgo, del quale il legislatore e, in generale, l'uomo autenticamente politico sono la copia, ha fatto sussistere l'universo, in modo che, nella misura del possibile, in esso ogni cosa fosse buona e nulla fosse cattivo ${ }^{51}$. È, dunque, l'opera demiurgica, in ultima

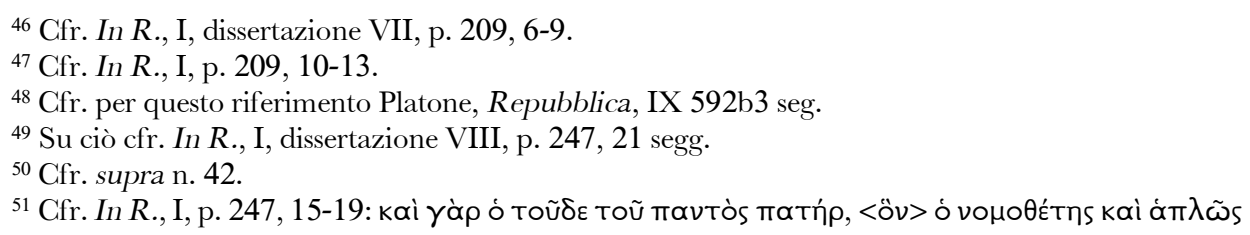


istanza, a costituire il fondamento originario di quell'armonia che nel mondo sensibile si delinea come imitazione ed immagine della perfetta e assoluta armonia che governa e permea il cosmo per intero. Inoltre nella città per il tramite del legislatore, imitatore del Demiurgo, uomini e donne hanno il compito di imitare, a loro volta, attraverso le virtù e con l'ausilio dell'educazione l'ordine divino dell'universo.

\section{La difesa della politeia platonica contro le critiche di Aristotele: l'unità come bene}

Come si è detto, la dissertazione XVII rappresenta una sorta di appendice al corpus di testi che costituiscono il commentario di Proclo alla Repubblica. In questa dissertazione, che ci è giunta mutila, egli ha come obiettivo quello di difendere la polis ideale di Platone dalle critiche che ad essa sono mosse Aristotele. Quest'ultimo, infatti, nel II libro della Politica, elabora un serrato attacco ai punti fondamentali del progetto politico platonico, ossia la comunione di averi, la comunanza di donne e di figli e, consequenzialmente, l'unità, più completa possibile, della città. In effetti proprio l'unità è secondo Aristotele il vero obiettivo che Platone si propone laddove espone le sue teorie sulla comunanza ${ }^{52}$.

Proclo affronta le critiche aristoteliche proprio alla luce della nozione di "unità", la quale, del resto, rappresenta il fondamentale concetto metafisico su cui poggia, come è noto, la gran parte dell'impianto teoretico della filosofia neoplatonica. In risposta alle critiche di Aristotele, il nostro commentatore elabora una generale e strenua difesa della nozione astratta di unità, anche se in questo contesto egli di fatto si riferisce specificamente all'unità in senso politico. La risposta di Proclo ad Aristotele sembra così fornire una sorta di "pretesto" per dimostrare che in ogni ambito del reale, compresa la politica, il bene coincide con l'unità: tale principio fondamentale va inteso alla luce della speculazione metafisico-teologica neoplatonica e in particolare a partire dall'assunto teoretico fondamentale secondo il quale il Bene si identifica con l'Uno, ovvero con il Principio Primissimo di tutto il reale. Da ciò, al contempo, consegue necessariamente che il male è originato da ciò che è opposto all'unità, vale a dire dalla molteplicità non ricondotta a ordine e unità.

Nella dissertazione XVII viene messo in luce come l'insieme delle critiche di Aristotele sia riconducibile al presupposto che nella polis non vi può essere una comunanza totale implicante una forma di unità integrale e assolutamente organica. La critica aristotelica al concetto di "unità" della polis, in effetti, muove dalla constatazione secondo cui non è possibile (áoúvatov) rendere effettivamente una la città e

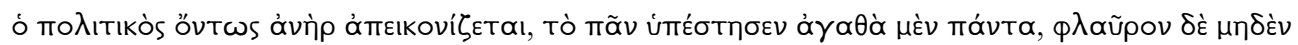

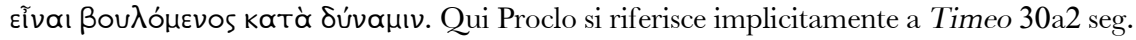

${ }^{52}$ Per un'analisi dell'argomentazione aristotelica si veda VegETTI 2002, in particolare pp. 183 segg. Si tenga altresì presente che la parte superstite della dissertazione XVII arriva solo a prendere in esame le obiezioni mosse da Aristotele nei primi tre capitoli del II libro della Politica. 
ciò non rappresenterebbe per essa neppure un bene: infatti per Aristotele la città è per sua natura una pluralità molteplice $(\pi \lambda \tilde{n} \theta 0 \varsigma)^{53}$. Alla luce di tale prospettiva, il punto di vista aristotelico viene sintetizzato nei seguenti termini: quanto più la città viene ad essere una, tanto più per essa verrà meno proprio il fatto stesso di essere una città ${ }^{54}$. Per Aristotele, infatti, la città non può essere ridotta a quell'unità ed omogeneità che sono proprie della famiglia e, in primo luogo, del singolo individuo. La città, come afferma Proclo per illustrare e chiarire la posizione aristotelica, verrebbe distrutta da un "livello eccessivo" di unità e, con ciò, quello che viene considerato come il sommo bene per essa, cioè che diventi quanto più è possibile una, le sarebbe assolutamente fatale. Se dunque l'unificare (Tò Éví̧દıv) la città risulta per essa distruttivo, mentre il bene di ciascuna cosa tende a conservare e non a distruggere ciò per cui è bene, l'unica conclusione possibile è che l'unificazione della città non è in realtà un bene per essa, poiché ciò sarebbe di fatto contrario alla sua stessa natura, in quanto sarebbe come

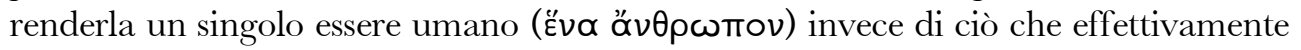
essa è, ossia una polis ${ }^{55}$.

Per rispondere a questa articolata obiezione, che, come si è detto, rappresenta per Proclo il punto centrale della critica aristotelica, il nostro commentatore precisa in quale senso deve essere intesa l'unità alla quale Platone, nel V libro della Repubblica, intende ricondurre la polis ideale. La questione viene affrontata da Proclo attraverso un'analisi ad un tempo logica e semantica del concetto di unità. In questo modo, egli, per certi versi, sembra voler sfidare e criticare Aristotele con le sue stesse armi e sul suo medesimo terreno. Proclo sottolinea come l'“unità" cui si riferisce Platone non sia quella intesa da Aristotele nella sua critica: non si deve intendere, spiega Proclo, l'unità come indivisibile e solo numerica; così concepita l'unità assumerebbe ruolo di materia ( $\lambda$ ó Yov ử $n_{5}$ ) in riferimento alla totalità della città ${ }^{56}$. Ciò equivale a dire che Aristotele, secondo Proclo, intende l'unità della polis di cui parla Platone, in senso puramente concreto e materiale: poiché la città è costituita da una molteplicità di individui, Aristotele avrebbe allora, in questo caso, ragione nel criticare la concezione platonica. In realtà, Platone intende riferirsi a quell'unità che è superiore alle parti (креĩtтov $\tau \tilde{\omega} v \mu \varepsilon^{\prime}(\omega v)$ e che è fonte di coesione per ciò che è intrinsecamente molteplice. In questo senso l'unità viene a rappresentare il superamento della divisione tra le parti e si delinea come una causa finale ( $\tau \varepsilon \lambda ı$ ıòv aítıov), grazie alla quale la molteplicità, secondo la prospettiva neoplatonica procliana, viene ricondotta ad un'armonica unitarietà omogenea. Non si tratta dunque di una qualche causa concomitante e mate-

${ }^{53} \mathrm{Su}$ ciò cfr. Aristotele, Politica, II 2, 1261 a13 segg.

${ }^{54} \mathrm{Su}$ ciò cfr. In R., II, dissertazione XVII, p. 361, 18 segg.

${ }^{55}$ Cfr. In R., II, p. 361, 20-29.

${ }^{56} \mathrm{Su}$ tutto ciò cfr. In R., II, p. 361, 29 segg. 


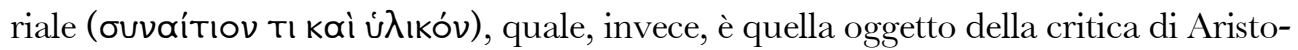
tele ${ }^{57}$.

La differenza che, in riferimento alla natura della polis, intercorre fra queste due differenti nozioni di unità - cioè tra quella alla quale si riferisce Platone e quella che è oggetto della critica di Aristotele - viene illustrata da Proclo ricorrendo all'esempio del "corpo", ampiamente utilizzato, come noto, nell'ambito del pensiero politico antico. Il corpo, spiega Proclo, è composto da molteplici parti, ciascuna delle quali è di per se stessa una unità: questo tipo di unità è tale nel senso di materia dell'intero (ஸ́s ü $\lambda \eta$ тoũ öגou). È in quest'ultima valenza che, a suo avviso, va inteso il concetto di unità cui si riferisce Aristotele nella sua critica. Invece la forma di unità che è per eccellenza tale è quella che mantiene insieme la totalità del corpo; a prescindere dalla specifica natura di questa unità, essa per Proclo svolge una funzione fondamentale, in quanto è in grado di ricondurre un'indistinta molteplicità di entità individuali all'armonia ed omogeneità proprie di un insieme unitario e ordinato. È proprio quest'ultima forma

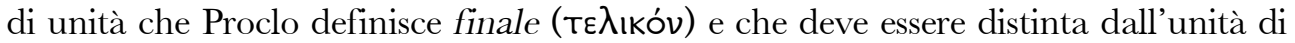
natura materiale (ú $\lambda_{\text {IKóv }}$ ) alla quale fa invece riferimento Aristotele nella sua critica ${ }^{58}$.

L'unità finale, in virtù della quale ciò che è molteplice viene a formare un insieme unitario e armonicamente strutturato, è ricondotta da Proclo al Bene in sé ( $\propto$ ưtò tò óy $\alpha \theta o ́ v)$, in virtù del quale e a partire dal quale tutte le cose hanno la loro unifica-

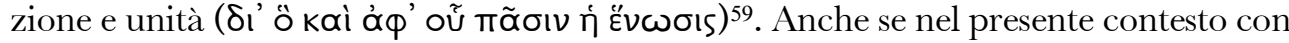
l'espressione "Bene in sé" il nostro commentatore forse non intende riferirsi direttamente al Bene-Uno (il Primissimo Principio assolutamente trascendente), bensì alla Forma intelligibile del Bene che, secondo la prospettiva procliana, si delinea come principio di perfezione e di unità, è evidente che qui Proclo intende comunque alludere all'identificazione neoplatonica fra Bene ed Uno. L'unità, entro questa prospettiva, si configura come bene per qualunque ente, nella misura in cui essa ne preserva l’integrità e la natura; all'opposto, invece, la molteplicità e la disomogeneità rappresentano i mali, proprio perché, invece di unire e armonizzare, sono causa di divisione e di disgregazione. Entro tale prospettiva Proclo interpreta anche il concetto di comu-

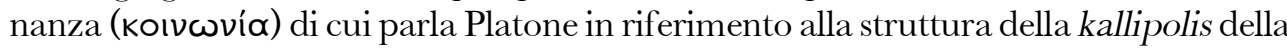
Repubblica: la koinonia ha proprio la funzione di unificare la città ed è causa di concordia e, perciò, mantiene la città libera dal pericolo delle sedizioni ${ }^{60}$.

${ }^{57}$ Per quanto qui affermato cfr. In R., II, pp. 361, 29-362, 6.

${ }^{58}$ Sull'esempio del corpo e sulla distinzione tra unità finale e unità materiale in riferimento all'argomentazione procliana in difesa di Platone cfr. In R., II, p. 362, 6-11.

${ }^{59}$ Cfr. In R., II, p. 362, 11-14. Sul tema centrale dell'"unità finale" rispetto a quella "materiale" nella dissertazione XVII si veda NARBOnNe 2003. Sulla dissertazione XVII di Proclo si veda anche lo studio di STALLEY 1995. Sulla critica che Proclo muove ad Aristotele per aver confuso l'unità "materiale" con quella "finale" si veda inoltre STALLEY 1999, in particolare pp. 43 segg.

${ }^{60}$ Cfr. In R., II, p. 362, 15 segg. 
Partendo da tali assunti, Proclo passa ad analizzare le tre ulteriori critiche che Aristotele muove a Platone:

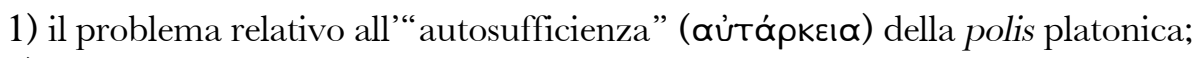

2) la critica all'abolizione della proprietà privata in connessione al problema dell'autosufficienza della città;

3) le implicazioni negative della "comunanza" di figli, donne e proprietà.

Proclo risponde ad ogni singolo punto ricorrendo sempre alla medesima strategia argomentativa fondata sulla superiorità, in senso metafisico-assiologico, di ciò che è uno ed unitario rispetto a ciò che è molteplice e dunque soggetto intrinsecamente a divisione.

Il problema dell'autosufficienza della polis viene posto da Aristotele, secondo la parafrasi che ne propone Proclo, nei seguenti termini: il fatto di unificare eccessivamente la città comporta l'annientamento dell'autosufficienza che essa deve possedere $^{61}$. In effetti, continua il nostro commentatore esponendo fedelmente il punto di

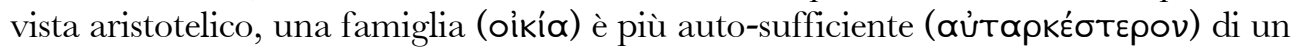

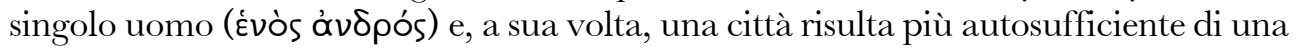
famiglia. Quindi secondo Aristotele, chiarisce Proclo, se ciò che è in misura minore un'unità è più autosufficiente, come appunto una città rispetto a una famiglia e una famiglia rispetto a un singolo uomo, ne consegue che, in modo inversamente proporzionale, quanto maggiore è l'unità tanto minore in essa sarà l'autosufficienza ${ }^{62}$. Proclo risponde a questa obiezione riprendendo la distinzione fra i due diversi modi di intendere l'unità: da un lato l'unità materiale che è indivisibile (ớtopov) in quanto è connotata in senso individuale, dall'altro quella finale che garantisce armonica unità a ogni realtà intrinsecamente composita. Anche in questo caso, chiarisce Proclo, Aristotele sbaglia poiché egli critica il progetto politico di Platone sulla base di una nozione di unità non corrispondente a quella cui si intende riferire Platone. Se infatti si considera l'intera questione a partire dalla prospettiva di quella che è autenticamente unità, la quale si identifica con ciò che è perfetto, in quanto ha di mira l'unità dell'intero, si deve concludere che la città è in misura maggiore unità rispetto al singolo individuo e che al contempo essa risulta in misura maggiore autosufficiente ${ }^{63}$. Secondo Proclo, dunque, Aristotele è in errore poiché egli con la sua argomentazione separa e distingue

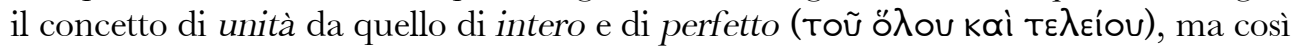
facendo, finisce per considerare l'unità come parte, dunque come qualcosa di intrin-

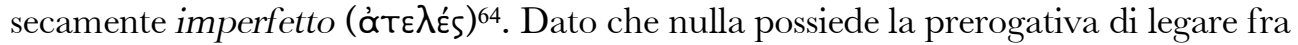
loro gli enti e di tenerli uniti insieme allo stesso modo del bene (ćs tò áyâóv), esso

${ }^{61}$ Cfr. In R., II, p. 362, 25 seg. Proclo qui si riferisce a quanto Aristotele afferma in Politica, II 2, 1261 b $10-15$.

${ }^{62}$ Cfr. In R., II, p. 362, 27 segg.

${ }^{63} \mathrm{Su}$ ciò cfr. In R., II, p. 363, $10 \mathrm{seg}$.

${ }^{64}$ Cfr. In R., II, p. 363, 15-17. 


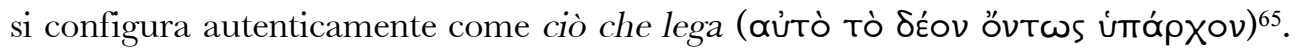
La città, dunque, come tutto ciò che partecipa in misura maggiore del bene, è più autosufficiente del singolo individuo in quanto essa partecipa in misura maggiore del bene e, di conseguenza, di ciò che è autenticamente unità: infatti il carattere dell'autosufficienza è una componente ( бтоıхẼ̃ov) del bene ${ }^{66}$. Questa è la conclusione di Proclo in risposta alla critica aristotelica sulla non-autosufficienza della polis platonica. È evidente che anche in questo contesto il nostro commentatore sviluppa la sua argomentazione alla luce della prospettiva metafisica neoplatonica, secondo la quale l'unità, in quanto deriva e dipende dall'Uno-Bene, è di per se stessa garanzia dell'armonia di qualsiasi entità intrinsecamente caratterizzata da composizione. Proprio nella misura in cui la città è unita, essa riflette in sé l'armonia e l'ordine che, come si è più volte ribadito, permeano, secondo la filosofia neoplatonica, i vari livelli entro cui si articola il reale. Da questo punto di vista il discorso procliano è in perfetta sintonia con quanto è affermato nelle Dissertazioni VII e VIII a proposito dell'analogia tra anima e città.

Ancora una volta, nella prospettiva esegetica procliana si delinea un rapporto perfettamente armonico tra anima-città e città-Tutto. È proprio sulla base di tale concezione di fondo che nella Dissertazione XVII Proclo afferma che, come la città è più autosufficiente del singolo individuo, così l'universo è più autosufficiente di ciascuna delle componenti che si trovano in esso ${ }^{67}$. La città (microcosmo) e l'universo (macrocosmo) partecipano infatti in misura maggiore dell' unità-bene rispetto alle loro componenti. In base a tale prospettiva l'unità e ciò che è autosufficiente vengono a coincidere. Allo stesso modo, il bene si configura a sua volta come unità e l'unità si delinea come bene ${ }^{68}$, proprio in base al presupposto dell'identificazione metafisica originaria fra il Bene e l'Uno. In questo modo la difesa della città ideale viene ricondotta da Proclo al principio fondamentale della concezione metafisico-teologica propria del neoplatonismo. Anche in questo contesto, dunque, emerge con chiara evidenza la coerenza sistemico-esegetica del nostro commentatore.

Per rispondere alla critica aristotelica relativa all'abolizione della proprietà privata, Proclo sottolinea come sia proprio in virtù dell'abolizione della proprietà e dei vincoli familiari privati che il bene della città viene a coincidere anche con quello del singolo individuo, in quanto quest'ultimo risulta così completamente assorbito nella collettività, allo stesso modo in cui la parte - per fare ricorso ad un esempio, come si è visto, caro a Proclo e in generale agli autori neoplatonici - è perfettamente compresa nell'intero. In tale maniera il singolo individuo ritiene di possedere come unico bene quello

\footnotetext{
${ }^{65}$ Cfr. In R., II, p. 363, 20 segg.

${ }^{66}$ Cfr. In R., II, p. 363, 26 segg.

${ }^{67}$ Cfr. In R., II, p. 364, 15 seg.

${ }^{68}$ Cfr. In R., II, p. 364, 21 segg.
} 
che è tale anche per la città ${ }^{69}$.

Dunque nella prospettiva procliana, come del resto in quella platonica, la comunanza di donne, figli ed averi - che nella concezione di Platone, occorre ricordare, riguarda solo le prime due classi, vale a dire quella dei filosofi-governanti e quella del gruppo militare - garantisce l'unità strutturale della città che in questo modo si delinea effettivamente come un "tutto" perfettamente armonico e omogeneo. Quest'armonia unitaria fa sì che tutti quanti, nella misura del possibile, abbiano un'unica e medesima

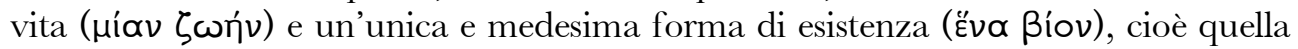
che è propria della città ${ }^{70}$. La koinonia insita nella città ideale rende assolutamente unitaria la vita dell'intera comunità, al punto che l'esistenza del singolo individuo viene a coincidere a tutti gli effetti con quella della città: in sostanza, si tratta nuovamente del medesimo rapporto che lega in modo pressoché inscindibile la parte con il tutto.

A questo punto, Proclo passa infine ad analizzare la critica che Aristotele rivolge alla comunanza di donne, di figli e di averi, secondo la quale gli esseri umani non si curerebbero delle cose che sono in comune, bensì solo delle loro personali proprietà: ciò per Aristotele implica, come conseguenza dell'abolizione della dimensione e del possesso privati, che tutti trascurino tutte le cose proprio perché sono considerate in comune $^{71}$. Purtroppo quest'ultima parte della Dissertazione XVII è assai lacunosa. È tuttavia possibile ricostruire almeno parzialmente il senso della risposta di Proclo contro questa critica di Aristotele. Sembrerebbe che Proclo basi la sua argomentazione sulla particolare educazione cui devono essere sottoposti tutti i cittadini nella polis platonica $^{72}$. L'iter educativo riservato da Platone ai cittadini della polis ideale, agli occhi del nostro commentatore, sembrerebbe così rappresentare lo strumento in virtù del quale ciascun individuo viene educato su un aspetto fondamentale e decisivo per l'integrità e l'unità complessiva della polis, cioè il non considerare nulla di proprio interesse, se questo differisce dal bene della città. Ciò significa che i membri della città ideale, allevati e educati secondo l'iter platonico, devono vivere anteponendo l'interesse comune, cioè della polis, a quello privato: in tal modo i cittadini sono portati a disinteressarsi del proprio personale vantaggio e a occuparsi solo ed esclusivamente del bene della comunità. Come sottolinea Proclo, il disprezzo dell'interesse privato farà rivolgere verso la totalità, mentre la tensione verso la totalità, a sua volta, farà rivolgere verso l'unità ${ }^{73}$. Si tratta ancora una volta della trasposizione in ambito eticopolitico del principio fondamentale su cui si regge l'intera metafisica neoplatonica: la

${ }^{69} \mathrm{Su}$ ciò cfr. In R., II, p. 366, $27 \mathrm{seg}$.

${ }^{70}$ Cfr. In R., II, p. 367, 8 seg.

${ }^{71} \mathrm{Su}$ questa critica cfr. Aristotele, Politica, II 3, 1261, b 32 segg. Per la sintesi procliana di tale critica cfr. In R., II, p. 367, 14 segg.

${ }^{72} \mathrm{Su}$ ciò cfr. In R., II, p. 367, 17 segg.

${ }^{73} \mathrm{Su}$ ciò cfr. In R., II, p. 368, 8-14. 
totalità e l'unità sono assiologicamente superiori a ciò che è molteplice, frammentario e particolare.

Il concetto di unità in senso finalistico viene così a rappresentare per il nostro commentatore il fondamento "politico-metafisico" su cui si regge la polis ideale di Platone. Anche se gli esseri umani tendono, come afferma lo Stagirita, a prendersi cura del loro interesse personale e a trascurare quello comune, ciò non significa - sembra argomentare Proclo - che tale condizione sia immodificabile: il superamento della dimensione dell'individuale e del particolare viene a rappresentare l'unica possibilità per rendere la città, intrinsecamente caratterizzata dalla molteplicità, un insieme unitario e armonico. In essa possono così rispecchiarsi quell'armonia e quella perfetta integrità strutturale che permeano, governano e mantengono unito l'universo, pur nelle sue molteplici articolazioni. La città ideale di Platone si delinea così come la migliore delle città proprio perché in essa si riflette la perfetta unità che caratterizza la natura del Tutto.

Per mettere ulteriormente in luce l'inconsistenza delle critiche aristoteliche, Proclo, nell'ultima pagina della parte di testo della dissertazione XVII che è giunta sino a noi, parafrasa il celeberrimo passo del libro X dell'Etica Nicomachea ove viene affermato da Aristotele che, a prescindere da quanto dicono i più, bisogna rendersi, per quanto è possibile, immortali ${ }^{74}$. Come ha ben messo in luce Stalley nel commento alla sua traduzione della dissertazione XVII ${ }^{75}$, in tal modo Proclo intende muovere una decisiva obiezione ad Aristotele: quest'ultimo, allorché espone le sue critiche alla polis platonica, parte dall'assunto che la vita felice va ricercata nell'ambito della proprietà privata e delle relazioni con gli altri privati cittadini; tuttavia proprio alla fine dell'Etica Nicomachea egli afferma che una vita veramente felice consiste nella contemplazione filosofica in virtù della quale l'esistenza umana si rende immortale ${ }^{76}$, cioè si fa simile a quella divina. Dunque sarebbe lo stesso Aristotele, in base all'osservazione procliana, che invita a superare ciò che appare come connaturato all'indole umana, ad esempio, per l'appunto, la cura dell'interesse personale e privato. D'altra parte, nell'interpretazione di Proclo, chi è stato allevato secondo il percorso educativo delineato da Platone nella Repubblica è in grado di oltrepassare la dimensione del privato e del particolare, per rivolgersi all'interesse comune della città. Proclo sembrerebbe così voler sottolineare che i cittadini della polis ideale di Platone, in virtù della loro tensione verso la totalità, a cui fa eco il disprezzo per l'interesse personale, si rendono immortali e dunque simili agli dèi. Si tratta forse di un accenno a un tema fondamentale per l'intera tradizione neoplatonica, vale a dire quello della ónoíwoıs $\theta \varepsilon \tilde{\omega}$, l'assimilazione a dio. Stando però a quanto ci rimane di questo testo procliano, non è possibile affermare

${ }^{74}$ Cfr. Aristotele, Etica Nicomachea, X 7, 1177 b 31 segg. Per la parafrasi procliana di tale passo cfr. In R., II, p. 368, 4 segg. Sul tema del rendersi immortali nella filosofia greca si veda VEGETTI 2001.

${ }^{75}$ Cfr. Stalley 1995, p. 149.

${ }^{76}$ Per inciso vale la pena sottolineare che nella parafrasi di Proclo, si legge $\alpha \dot{\pi} \alpha \theta \alpha v \alpha \tau i ́ \zeta \varepsilon ı v$, mentre

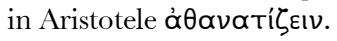


ciò con certezza.

Comunque, anche dall'esame delle risposte di Proclo alle critiche aristoteliche emerge come egli tenda a ricondurre ogni questione, anche di carattere propriamente etico-politico, ai presupposti teoretici essenziali della metafisica e della teologia neoplatoniche.

4. Il senso complessivo dell'interpretazione procliana della Repubblica

Alla luce delle analisi qui condotte sui temi principali affrontati nel corpus di testi che costituisce il Commento alla Repubblica di Proclo, emerge una chiara ed unitaria visione d'insieme: l'intero e complessivo impianto esegetico procliano appare incentrato sulla relazione originaria tra la nozione di unità e quella di tutto e sull'identificazione del Bene con l'Uno. Tali presupposti concettuali, secondo la prospettiva ermeneutica procliana, permeano, in modo ed in misura di volta in volta differenti, $\mathrm{i}$ diversi ambiti di indagine entro i quali si articola questo imponente dialogo di Platone: dall'etica alla politica, dalla metafisica alla teologia. Quest'ultima, in particolare, si delinea come il centro focale in dipendenza ed alla luce del quale vengono affrontate tutte le altre tematiche filosofiche. In questo senso nell'esegesi procliana della Repubblica si riflettono in modo assai preciso e nitido i contorni e le caratteristiche fondamentali del complesso ed articolato sistema metafisico-teologico che contraddistingue, in modo essenziale e specifico, la tradizione filosofica tardo-neoplatonica di cui Proclo è senza dubbio uno tra i massimi rappresentati, forse il più grande e significativo.

Alla luce dell'interpretazione procliana è possibile altresì ricavare alcune considerazioni di carattere generale sul modo in cui anche gli altri autori della tradizione tardo-neoplatonica potevano aver inteso la Repubblica nel suo complessivo significato: essa si configurava, per molti aspetti, come una summa paradigmatica e generale della dottrina platonica in tutte le sue diverse articolazioni, ossia etica, politica, epistemologia, metafisica e teologia. Tale dialogo, così, fu probabilmente considerato come un microcosmo rispecchiante nella sua complessità le strutture e le gerarchie del reale: una finestra, potremmo dire, che si apre sull'autentica natura (intrinsecamente molteplice e articolata, secondo la prospettiva neoplatonica) del Tutto.

Agli occhi di Proclo e, probabilmente, degli autori neoplatonici a lui precedenti, la Repubblica appariva, per la sua ampiezza e complessità, come un testo che consentiva di spaziare su svariate questioni filosofiche decisive ed essenziali, ma troppo complesse per poter essere oggetto di una trattazione unitaria. Per un autore neoplatonico commentare parti della Repubblica poteva comunque fornire una via di accesso per giungere a quella oúvoభıs - ossia a quella visione complessiva, unitaria e sistematica dei vari livelli in cui si articola il reale - che costituiva uno degli obbiettivi fondamentali dell'intera riflessione neoplatonica. 


\section{Bibliografia}

Abbate, M. (1999), «Gli aspetti etico-politici della Repubblica nel commento di Proclo (dissertazioni VII/VIII e XI)», in M. Vegetti, M. Abbate (eds.), La Repubblica di Platone nella tradizione antica, Napoli, 207-218.

Abbate, M. (2003), «Il Bene nell'interpretazione di Plotino e di Proclo», in M. Vegetti (ed.), Platone. La Repubblica, V, Napoli, 625-678.

Abbate, M. (2004), «Proclo interprete della Repubblica», in M. Abbate (ed.), Proclo. Commento alla Repubblica (Dissertazioni I-V, VII-XII, XIV-XV, XVII), Milano.

Abbate, M. (2006), «Metaphysics and Theology as Methodological and Conceptual Paradigms in Proclus' Ethico-Political Theory», in M. Perkams, R.M. Piccione (eds.), Proklos. Methode, Seelenlehre, Metaphysik, Köln-Leiden-New York, 186-200.

Abbate, M. (2007), «L'interpretazione del Bene nella Dissertazione XI del Commento alla Repubblica di Proclo», in F.L. Lisi (ed.), The Ascent to Good, Sankt Augustin, 241-249.

Abbate, M. (2008), Il divino tra unità e molteplicità. Saggio sulla Teologia Platonica di Proclo, Alessandria.

Abbate, M. (2012), Tra esegesi e teologia. Studi sul Neoplatonismo, Milano-Udine.

Abbate, M. (2016), «La fonte originaria della luce intelligibile secondo l'ermeneutica neoplatonica», in M. Borriello, A.M. Vitale (eds.), Princeps philosophorum. Platone nell'Occidente tardo-antico, medievale e umanistico, Roma 2016, 53-65.

Coulter, A. (1976), The Literary Microcosm. Theories of Interpretation of the Later Neoplatonism, Leiden.

Dörrie, H., Baltes, M. (1993), Der Platonismus in der Antike, Bd. III. Der Platonismus im 2. und 3. Jahrhundert nach Christus. Bausteine 73-100, Stuttgart-Bad Cannstatt.

Gallavotti, C. (1971), «Intorno ai commentari di Proclo alla Repubblica», Bollettino del comitato per la preparazione dell'edizione nazionale dei classici greci e latini, 19, 41-54.

Gerson, L.P. (2016), «The 'Neoplatonic' Interpretation of Plato's Parmenides», The International Journal of the Platonic Tradition, X 1, 65-94.

Hadot, I. (1987), «Les introductions aux commentaires exégétiques chez les auteurs néo-platoniciens et les auteurs chrétiens», in E. Tardieu (éd.), Les règles de l'interprétation, Paris, 99-122.

Kroll, W. (ed.) (1899-1901), In Platonis Rem publicam commentarii, I-II, Leipzig (rist. Amsterdam 1965).

Mansfeld, J. (1994), Prolegomena. Questions to be settled before the study of an author, or a text, Leiden-New York-Köln.

Motta, A. (ed.) (2014), Prolegomeni alla filosofia di Platone, Roma.

Narbonne, J.-M. (2003), «De l'un matière à l'un forme. Le réponse de Proclus à la critique aristotélicienne de l'unité du politique dans la République de Platon (In Rem. II, 361368)», in J.-M. Narbonne, A. Reckermann (éds.), Pensées de l'Un dans la tradition métaphysique occidentale. Festschrift Werner Beierwaltes, Paris-Montréal, 3-25.

O’Meara, D.J. (1999), «Plato's Republic in the School of Iamblichus», in M. Vegetti, M. Abbate (eds.), La Repubblica di Platone nella tradizione antica, Napoli, 193-205.

O’Meara, D.J. (2003), Platonopolis. Platonic political Philosophy in late Antiquity, Oxford.

Pichler, R. (2006), Allegorese und Ethik bei Proklos. Untersuchungen zum Kommentar zu 
Platons Politeia, Berlin.

Sheppard, A. (1980), Studies on the $5^{\text {th }}$ and $6^{\text {th }}$ Essays of Proclus' Commentary on the Republic, Göttingen.

Stalley, R.F. (1995), «The Unity of the State, Plato, Aristotle and Proclus», Polis, 14, 129149.

Stalley, R.F. (1999), «Plato and Aristotle on Political Unity», in M. Vegetti, M. Abbate (eds.), La Repubblica di Platone nella tradizione antica, Napoli, 29-48.

Vegetti, M. (2001), «“Athanatizein”. Strategie di immortalità nel pensiero greco», Aut Aut, $304,69-80$.

Vegetti, M. (2002), «La critica aristotelica alla Repubblica di Platone nel secondo libro della Politica», in M. Migliori (ed.), Gigantomachia. Convergenze e differenze tra Platone e Aristotele, Brescia, 179-190.

Vegetti, M. (2009), «Un paradigma in cielo». Platone politico da Aristotele al Novecento, Roma.

Westerink, L.G. (ed.) (1990), Prolégoménes à la philosophie de Platon, Paris. 